\title{
Biorelevant dissolution testing of a weak base: Interlaboratory reproducibility and investigation of parameters controlling in vitro precipitation
}

Philippe Berben ${ }^{1}$, Lee Ashworth ${ }^{2}$, Stefania Beato ${ }^{3}$, Jan Bevernage ${ }^{4}$, Jean-Luc Bruel ${ }^{5}$, James Butler ${ }^{6}$, Jennifer Dressman ${ }^{7}$, Kerstin Schäfer ${ }^{8}$, Paul Hutchins ${ }^{9}$, Lukas Klumpp ${ }^{7}$, James Mann ${ }^{2}$, Janine Nicolai ${ }^{10}$, Krista Ojala ${ }^{11}$, Sanjaykumar Patel ${ }^{12}$, Sarah Powell ${ }^{2}$, Karin Rosenblatt ${ }^{13}$, Irena Tomaszewska $^{14}$, James Williams ${ }^{6}$, Patrick Augustijns ${ }^{1}$.

${ }^{1}$ Drug Delivery and Disposition, University of Leuven (Leuven, Belgium)

${ }^{2}$ Pharmaceutical Technology \& Development, AstraZeneca (Macclesfield, United Kingdom)

${ }^{3}$ Technical Research and Development, Novartis Pharma AG (Basel, Switzerland)

${ }^{4}$ Pharmaceutical Sciences, Janssen Pharmaceutica, Johnson \& Johnson (Beerse, Belgium)

${ }^{5}$ Research \& Development, Sanofi-Aventis (Vitry-sur-Seine, France)

${ }^{6}$ GlaxoSmithKline R\&D, Pharmaceutical Development \& Supply (Ware, UK)

${ }^{7}$ Institute of Pharmaceutical Technology, Goethe University (Frankfurt am Main, Germany)

${ }^{8}$ Pharmaceutical Development, Boehringer Ingelheim Pharma GmbH (Biberach an der Riß, Germany)

${ }^{9}$ Drug Product Science and Technology, Bristol-Myers Squibb (Moreton, UK)

${ }^{10}$ Chemical and Pharmaceutical Development, Bayer AG (Wuppertal, Germany)

${ }^{11}$ Pharmaceutical Sciences, Analytical Development, Orion Pharma (Turku, Finland)

${ }^{12}$ Analytical Sciences, MRL, Merck \& Co., Inc. (Kenilworth NJ, USA)

${ }^{13}$ Drug Product Development, AbbVie Deutschland GmbH \& Co. KG (Ludwigshafen, Germany)

${ }^{14}$ Analytical Research and Development, Pfizer Ltd. (Sandwich, UK)

Corresponding author:

Patrick Augustijns

Drug Delivery and Disposition, KU Leuven, Gasthuisberg O\&N II, Herestraat 49 - box 921, 3000 Leuven, Belgium

Tel: +3216330301 Fax: +3216330305

Patrick.augustijns@kuleuven.be 


\section{KEY WORDS}

32 Indinavir sulfate

33 Dissolution testing

34 USP 2 Apparatus

35 OrBiTo

36 Interlaboratory reproducibility

37 Supersaturation/precipitation

38

39

40

41

42

43

44

45

46

47

48

49

50

51

52

53

54

55

56

57

58

59

60

61 


\section{ABSTRACT}

Following a previous study which aimed to determine the interlaboratory reproducibility of biorelevant dissolution testing in the USP 2 apparatus for commercial formulations of two weak acids (ibuprofen and zafirlukast), this study attempts to determine the interlaboratory reproducibility using a similar protocol for a commercially available formulation of a weak base, indinavir. Fourteen partners including twelve industrial and two academic partners participated in this study. To ensure uniformity, all partners were provided with a standardized protocol to perform (i) a single medium dissolution test in fasted state simulated gastric and intestinal fluids (FaSSGF and FaSSIF, respectively) and (ii) a twostage dissolution experiment simulating gastrointestinal transfer. Optionally, partners could run a single-stage dissolution test in fed state simulated intestinal fluid (FeSSIF). For each dissolution test, one Crixivan ${ }^{\circledR}$ capsule (containing $400 \mathrm{mg}$ indinavir as its sulfate salt) was added as dose of interest.

For the single medium dissolution test in FaSSIF, all partners observed rapid release of indinavir resulting in supersaturated concentrations, followed by precipitation to equilibrium solubility. The degree and period of supersaturation varied among the participating laboratories. Average dissolution profiles in FeSSIF appeared to be highly reproducible with dissolved concentrations remaining lower than the thermodynamic solubility of indinavir in FeSSIF. For the two-stage dissolution test, most partners observed supersaturated concentrations in the intestinal compartment; two partners observed no supersaturation due to immediate precipitation. Given the fact that a high interlaboratory but low intralaboratory variability was observed when supersaturation/precipitation occurred, an undefined factor was hypothesized as a potential cause of the variability in precipitation. Hence, the impact of several experimental factors on the supersaturation and precipitation behavior of indinavir was investigated in a next step.

The investigation indicated that variability is likely attributable to a combination of factors, especially, the time elapsed between sampling and dilution of the sample with the mobile phase. Therefore, when 
0

designing a test in which supersaturation and precipitation is anticipated, stringent control of the test methodology, especially regarding sampling and dilution, is needed.

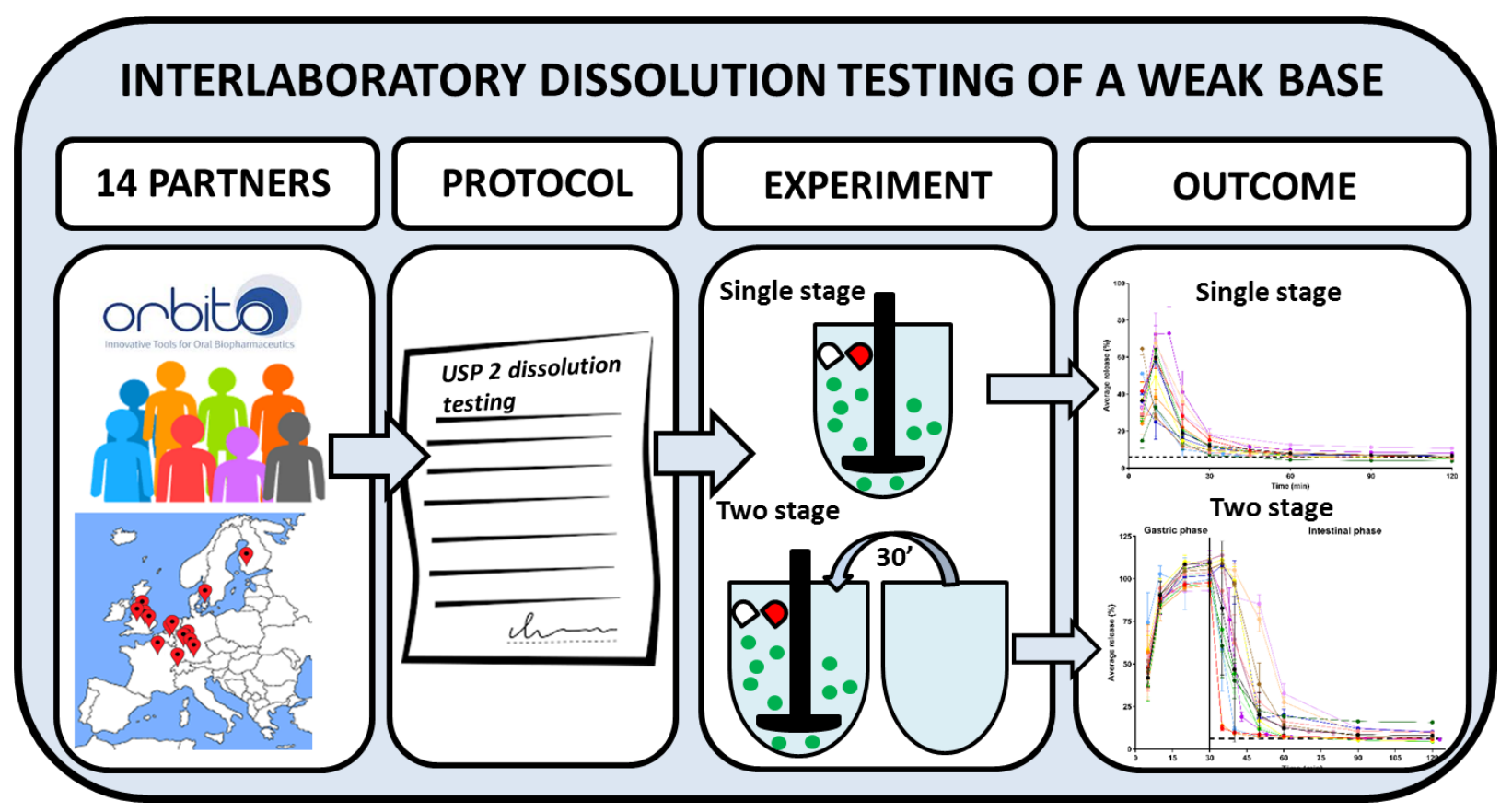


Despite dissolution traditionally being used for quality control (QC) purposes, the application of dissolution to predict the in vivo biopharmaceutical performance of oral dosage forms is gaining considerable interest [1]. For example, regulatory authorities like the European Medical Agency (EMA) advise the use of USP 1 and 2 dissolution test apparatus as a safe and reliable strategy for biowaiver testing of immediate release (IR) formulations of BCS class I and III drugs [2] since these dissolution tests are easy-to-handle and robust. Additionally, formulation scientists often use these dissolution tests during the development of new drug candidates in order to select the formulation strategy with the highest potential for reaching the market [3-5]. Although a multitude of intrinsic and extrinsic parameters may affect the dissolution profile, it is crucial that test results can be integrated into the drug development process with confidence, so the optimal formulation can be selected without compromising in vivo performance or reducing development timelines [6].

Recently, a study among 16 partners participating in the European project OrBiTo (Oral

Biopharmaceutics Tools) was performed to evaluate the interlaboratory reproducibility of dissolution testing in the paddle apparatus (USP 2) with biorelevant media; commercially available ibuprofen tablets and zafirlukast tablets were selected as formulations of interest [7]. For both drugs, a high degree of reproducibility was observed in terms of the dissolution profile for both a single medium and a two-stage dissolution test. Since both drugs are weak acids, release is slow in the acidic environment used in the first stage to represent the stomach but fast in the neutral medium used in the second stage to represent the small intestine; supersaturation and precipitation were neither expected nor observed. By contrast, the $\mathrm{pH}$-shift due to transfer from the stomach to the small intestine may trigger supersaturation of weak base drugs, which may result in precipitation. Under these circumstances, attaining reproducible results in a representative dissolution test is more challenging.

121 Over the past 15 years, several in vitro setups have been developed to evaluate supersaturation and 122 precipitation, ranging from relatively simple to rather complex tools. For example, Kostewicz et al. 
developed a simple transfer model by connecting two USP 2 vessels with a peristaltic pump for the prediction of intestinal supersaturation and precipitation [4]. On the other hand, the TNO intestinal model 1 (TIM-1) is a more advanced tool allowing high-quality prediction of the in vivo behavior of orally administered drugs [8]. Given the complexity of supersaturation and precipitation, multiple factors may affect this interplay in vivo including gastric emptying rate, gastrointestinal motility, permeability, the physicochemical properties of the drug, and the volume and composition of gastrointestinal fluids [9]. Since simple dissolution tests do not succeed to capture all these dynamic processes, it is highly advised to combine in vitro data with physiologically-based pharmacokinetic (PBPK) models for a more accurate prediction of the PK profile of oral dosage forms $[10,11]$. In the case of weak bases, these software programs typically rely on the input from in vitro supersaturation and precipitation experiments in combination with human physiology data and physicochemical properties of the drug. As such, reliability of the in vitro methods must be guaranteed to accurately predict the in vivo drug behavior.

Using the same approach as the previous study in which the interlaboratory reproducibility of two weak acids was assessed, the current study aimed to evaluate the robustness of a biorelevant dissolution test in the USP 2 apparatus for a weak base. Participating partners were required to perform (i) a single medium dissolution test in FaSSGF and FaSSIF (and optionally in FeSSIF) and (ii) a two-stage dissolution test simulating the gastrointestinal transfer. Crixivan ${ }^{\circledR}$, containing indinavir sulfate, was selected as the formulation of interest since the gastrointestinal behavior of indinavir has been recently elucidated by administering Crixivan $^{\circledR}$ capsules to human healthy volunteers in the fasted state [12]. Fourteen partners participated in this cross-laboratory study including AbbVie Deutschland GmbH \& Co. KG, AstraZeneca, Bayer AG, Boehringer Ingelheim Pharma GmbH, BristolMyers Squibb Pty. Ltd., GlaxoSmithKline, Goethe University, Janssen Pharmaceutica, KU Leuven, Merck \& Co. Inc., Novartis Pharma AG, Orion Pharma, Pfizer Ltd. and Sanofi-Aventis. 


\section{MATERIALS AND METHODS}

\subsection{Selection of the formulation of interest}

Indinavir is a weak base with pKa values of 3.7 and 5.9. While the solubility of indinavir is very high in an acidic environment $(>99.4 \mathrm{~g} / \mathrm{L}$ at $\mathrm{pH}<3.5)$, its solubility in a neutral environment is relatively low $(0.048 \mathrm{~g} / \mathrm{L}$ in FaSSIF, pH 6.5) $[13,14]$. According to the criteria of the Biopharmaceutics Classification System (BCS), indinavir has been reported to be either a class II (low solubility, high permeability) or class IV (low solubility, low permeability) drug, as the intestinal permeability of indinavir sits on the border of the permeability criterion of the BCS. Since indinavir shows poor aqueous solubility, the drug is formulated as a sulfate salt (Crixivan ${ }^{\circledR}, 400 \mathrm{mg}$ indinavir) for which superior bioavailability has been demonstrated compared to the free base [15]. Furthermore, a clinical trial has been recently performed to elucidate the intraluminal behavior of Crixivan ${ }^{\circledR}[12]$.

\subsection{Chemicals and media}

All participating partners were initially provided with a small amount of pure indinavir sulfate and with commercially available capsules of indinavir sulfate (Crixivan ${ }^{\circledR}$ ) originating from the same batch (L006343). Subsequent investigations of the source of variability were conducted using a different capsule batch (N006110). Each partner was expected to purchase the remaining material and chemicals independently implying that, most likely, different filters and different batches of FaSSIF/FeSSIF/FaSSGF powder were used.

Biorelevant media were prepared according to instructions provided by Biorelevant.com. Fasted state simulated gastric fluid (FaSSGF) was obtained by adding FaSSIF/FeSSIF/FaSSGF powder $(0.06 \mathrm{mg} / \mathrm{mL})$ to blank FaSSGF $(1.99 \mathrm{mg} / \mathrm{mL} \mathrm{NaCl}$, adjusted to $\mathrm{pH} 1.6$ with $1 \mathrm{M} \mathrm{HCl})$. Fasted state simulated intestinal fluid (FaSSIF) was prepared by dissolving FaSSIF/FeSSIF/FaSSGF powder $(2.24 \mathrm{mg} / \mathrm{mL})$ in blank FaSSIF (3.95 mg/mL NaH $2 \mathrm{PO}_{4} \cdot \mathrm{H}_{2} \mathrm{O}, 6.19 \mathrm{mg} / \mathrm{mL} \mathrm{NaCl}, 0.42 \mathrm{mg} / \mathrm{mL} \mathrm{NaOH}$, adjusted to $\mathrm{pH}$ 6.5) while doubleconcentrated FaSSIF (2x FaSSIF) was obtained by dissolving double amounts of $\mathrm{NaH}_{2} \mathrm{PO}_{4} \cdot \mathrm{H}_{2} \mathrm{O}, \mathrm{NaCl}$, $\mathrm{NaOH}$ and FaSSIF/FeSSIF/FaSSGF powder compared to normal FaSSIF, and adjusted to $\mathrm{pH} 7.5$ with $2 \mathrm{M}$ 
of $\mathrm{NaOH}$, so that, after addition of $250 \mathrm{~mL}$ of $2 x$ FaSSIF to $250 \mathrm{~mL}$ of FaSSGF, the final pH amounts to 6.5. Fed state simulated intestinal fluid (FeSSIF) was prepared by dissolving FaSSIF/FeSSIF/FaSSGF powder $(11.2 \mathrm{mg} / \mathrm{mL})$ in blank FeSSIF $(4.04 \mathrm{mg} / \mathrm{mL} \mathrm{NaOH}, 8.65 \mathrm{mg} / \mathrm{mL}$ glacial acetic acid, $11.87 \mathrm{mg} / \mathrm{mL}$ $\mathrm{NaCl}$, adjusted to $\mathrm{pH}$ 5.0). Media were equilibrated prior to use to allow for micelle particle size stabilization and were considered to be stable for $48 \mathrm{~h}$ at room temperature [16].

\subsection{Protocols to perform dissolution tests}

All partners were asked to perform two types of dissolution tests with Crixivan ${ }^{\circledR}$ including (i) a single medium dissolution test in FaSSGF and FaSSIF (and optionally in FeSSIF) and (ii) a two-stage dissolution test simulating the gastrointestinal transfer. Below, the protocols for the single medium (cfr. 2.3.1.) and two-stage (cfr. 2.3.2.) dissolution test are described as they were shared with the participating partners.

\subsubsection{Single-stage biorelevant dissolution}

Single-stage biorelevant dissolution tests were performed according to the following standardized protocol:

- Apparatus: the USP 2 Apparatus (rotating paddle) was used with a paddle speed of $75 \mathrm{rpm}$, at $37^{\circ} \mathrm{C}$, using a volume of $250 \mathrm{~mL}$ for FaSSGF, and $500 \mathrm{~mL}$ for FaSSIF and FeSSIF.

- Sampling time points: samples were taken after 5, 10, 20 and $30 \mathrm{~min}$, and (if the product proved to dissolve slowly) every subsequent 30 min until a plateau was reached. Partners were asked to add extra early time point(s) if plateau was reached within 10 minutes or less.

- Dose: protocol instructions indicated to mimic the dose of interest in humans, even if this means adding multiple dose units to the vessel. In this case, dissolution experiments were performed using one capsule of Crixivan ${ }^{\circledR}$. Instructions were to run at least 3 replicates for each type of experiment; most partners ran six replicates. 
- Sampling and analysis: although sampling methodology was allowed to vary among the partners, it was recommended to use manual sampling via syringe $(5 \mathrm{~mL}$, from which an approximate $0.5 \mathrm{~mL}$ analysis sample was taken), followed by analysis using a method appropriate for the drug. To this end, the first $2-3 \mathrm{~mL}$ was passed through the filter and any excess of the sample in the syringe was to be returned to the vessel. Moreover, samples should be appropriately diluted with mobile phase and analyzed on the same day, unless stability could be confirmed over a longer period. Other sampling and analysis methods (e.g. semi-automated sampling, UV detection) were allowed at the discretion of the partner provided they were compatible with the sample properties. Issues to be considered included sample precipitation prior to analysis during semi-automated sampling and the potential impact of variable background UV absorbance if using UV detection without HPLC.

- Filtration: to separate undissolved particles from dissolved particles, a suitable filter should be selected, with a check for adsorption of the drug onto the filter. It was not allowed that the adsorbed fraction exceeded $2 \%$. If a larger fraction was adsorbed, then this should be clearly stated in the report. Typically, a PTFE, GHP or glass fiber filter was used (pore size $0.45 \mu \mathrm{m}$, diameter $25 \mathrm{~mm}$ ). In addition, if there was an excess of undissolved excipient/drug present, it was advised to use a pre-filter to avoid filter clogging.

- Product specific modifications to the method: if supersaturation is likely in the samples taken, then samples should be diluted immediately after sampling to avoid (further) precipitation.

The partners were also advised that, when using a volume of $250 \mathrm{~mL}$ in a standard $1 \mathrm{~L}$ dissolution vessel, the use of bathless dissolution vessels should be avoided due to heating zone issues. Additionally, partners were informed that the paddles are very close to the medium surface at this (lower than typical) volume implying that sampling challenges might result in some variability. 


\subsubsection{Two-stage biorelevant dissolution (representing the gastrointestinal transfer)} Two-stage biorelevant dissolution tests were performed according to the following standardized protocol:

- Apparatus: the USP 2 Apparatus (rotating paddle) was used with a paddle speed of $75 \mathrm{rpm}$, at $37^{\circ} \mathrm{C}$, using an initial volume of $250 \mathrm{~mL}$ for FaSSGF ( $\left.\mathrm{pH} 1.6\right)$ for $30 \mathrm{~min}$. Subsequently, $250 \mathrm{~mL}$ of $2 x$ FaSSIF ( $\mathrm{pH} 7.5$ ) was added to end up with a final volume of $500 \mathrm{~mL}$ of normal FaSSIF (pH 6.5).

- Sampling time points: during the gastric stage, samples were taken after 5, 10, 20 and 30 min. Following the addition of 2x FaSSIF, additional samples were taken after 35, 40, 50 and $60 \mathrm{~min}$, and (if slowly dissolving) every subsequent 30 min until a plateau was reached. It was recommended to add extra early time point(s) if the plateau was reached within 10 min or less after addition of $2 x$ FaSSIF.

- Dose selection, sampling, filtration and analysis were performed as described for the single-stage dissolution test. Any product-specific modifications to the methodology were expected to be clearly reported.

\subsection{Variations from the protocol}

As the protocols provided some flexibility in the experimental design, there were several instances in which variations on the protocol occurred or a partner chose to explore some of the parameters that were not explicitly specified in the protocol. For instance, although the protocol intended that the media should be preheated before use, one partner ran the studies without preheating the media. Despite the sampling time points being clearly defined in the protocol, some partners took samples at additional time points or stopped the experiment earlier than instructed. In addition, one partner used a semi-automated UV system, meaning that indinavir concentrations were measured online, without a dilution step. Furthermore, the protocol clearly stated that if supersaturation is likely to occur (which is the case for indinavir), samples should be diluted immediately after sampling to stop ongoing 
precipitation. Nevertheless, the time interval between taking and diluting the samples appeared highly

248 variable among the different partners (as discussed below).

\subsection{Analytical methods}

250 To determine the concentrations of indinavir, most partners developed an in-house HPLC or UPLC

251 method whilst one partner used a semi-automated UV system. Exact details of the analytical methods

252 from the individual partners can be obtained upon request. A typical method for the HPLC analysis of 253 indinavir is shown in Table 1.

254 Table 1. Typical conditions for the HPLC analysis of indinavir

\begin{tabular}{cc} 
HPLC & Waters ${ }^{\mathrm{TM}} 600$ Controller \\
Column & Novapak C18 $(4 \mu \mathrm{m}, 8 \mathrm{~mm} \times 100 \mathrm{~mm}$, Waters $)$ \\
Mobile phase & Acetate buffer $(25 \mathrm{mM}) /$ methanol $(25 / 75)$ \\
pH mobile phase & 3.5 \\
Flow rate & $1 \mathrm{~mL} / \mathrm{min}$ \\
Detection & UV: $260 \mathrm{~nm}$ or Fluo: Ex: $256 \mathrm{~nm} / \mathrm{Em}: 290 \mathrm{~nm}$ \\
Injection volume & $50 \mu \mathrm{L}$ \\
Retention time & $5.1 \mathrm{~min}$ \\
Analysis time & $8 \mathrm{~min}$ \\
\hline
\end{tabular}




\subsection{Single-stage dissolution profiles}

The average dissolution profiles of indinavir in FaSSGF from all partners are depicted in Figure 3 as the first 30 min of the two-stage dissolution test reflect the dissolved fraction in FaSSGF. In this acidic environment, a fast dissolution of Crixivan ${ }^{\circledR}$ was observed whereby almost $50 \%$ of the drug was dissolved after $5 \mathrm{~min}$; in most cases, over $90 \%$ of indinavir was dissolved within 10 min. Furthermore, dissolved concentrations of indinavir were highly reproducible among the different participating partners (e.g. RSD $=5.7 \%$ after $30 \mathrm{~min}$ ). Most likely, the fast and complete dissolution of indinavir are the result of its excellent solubility in the acidic environment $(>99.4 \mathrm{~g} / \mathrm{L}$ at $\mathrm{pH}<3.5)$ and the immediate release properties of the formulation. Similar behavior has been demonstrated in the fasted stomach of human healthy volunteers following the oral administration of a single capsule of Crixivan ${ }^{\circledR}$ [12].

In contrast to the gastric medium, indinavir is predominantly unionized at the $\mathrm{pH}$ of the intestinal environment. Together with the partition coefficient $\left(\log D_{p H} 6.52 .4\right)$, this makes it likely that indinavir shows a high affinity for colloidal structures such as bile salts and phospholipids, as previously demonstrated by Holmstock et al. [17]. Biorelevant media, containing these colloidal structures, are therefore recommended for dissolution testing of indinavir rather than simple pharmacopeial buffers. As a result, partners were asked to perform a dissolution test in FaSSIF and optionally in FeSSIF. Figure 1 depicts the average dissolution profiles from all partners in FaSSIF. Every partner observed concentrations of indinavir higher than its thermodynamic solubility in FaSSIF (dotted line), indicating that the sulfate salt of indinavir enables generation of supersaturation during dissolution in FaSSIF. In all cases, the supersaturated indinavir levels induced precipitation. As a wide range of maximum concentrations $\left(C_{\max }\right)$ were observed (maximum dissolved fraction ranging from $29.1 \%-72.9 \%$ ), the degree of supersaturation was highly variable among the different participating laboratories. In addition, most partners observed a $\mathrm{C}_{\max }$ after $10 \mathrm{~min}$; however, four partners measured a maximum concentration of indinavir at the first sampling time point (5 $\mathrm{min})$. 


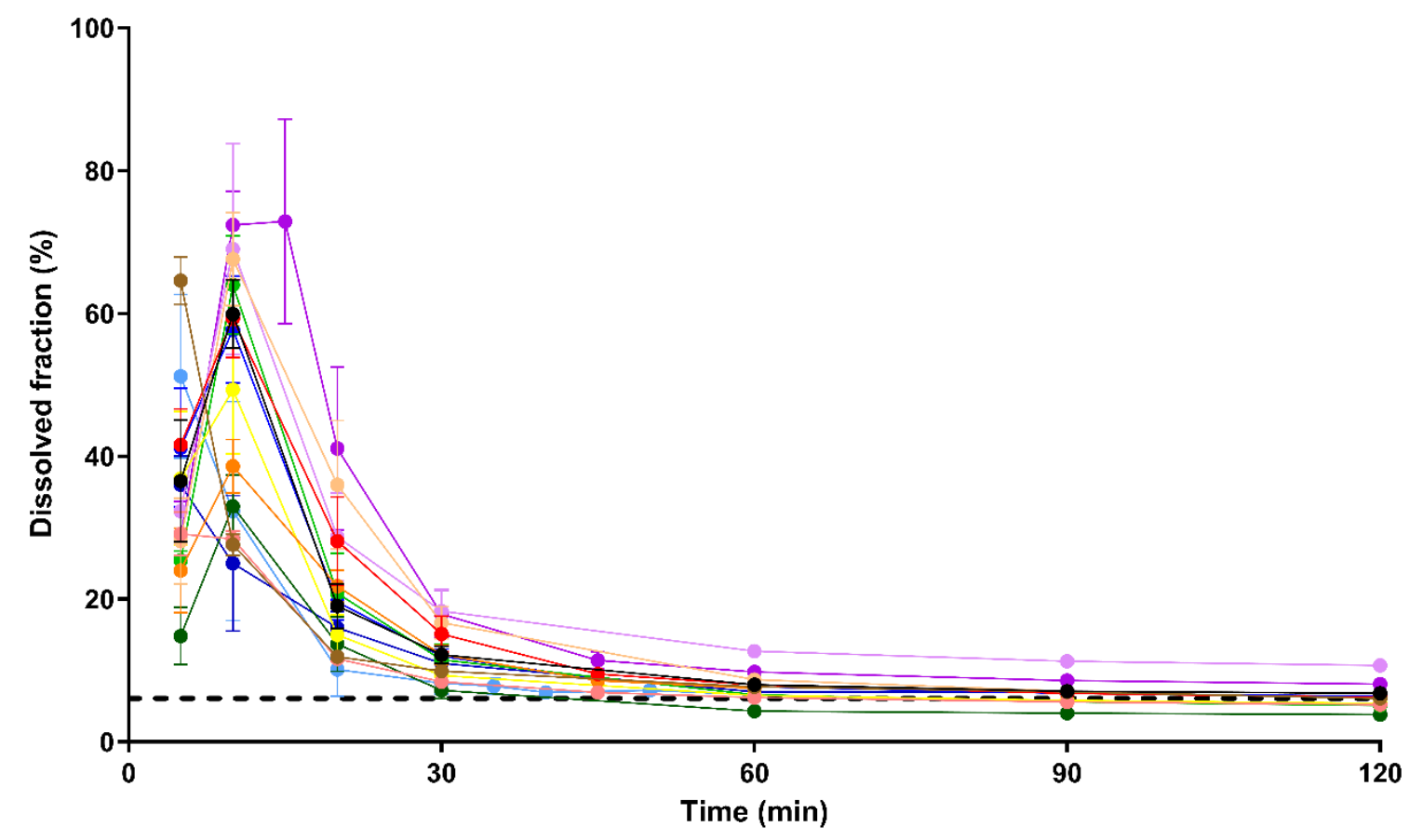

285

Figure 1: Dissolution of indinavir from Crixivan ${ }^{\circledR} 400 \mathrm{mg}$ capsules in FaSSIF. Each colored curve represents the results from one partner (mean $\pm S D, n=3-6$ ). The dotted line represents the dissolved fraction corresponding to the thermodynamic solubility of indinavir in FaSSIF.

As the dissolution test in FeSSIF was optional, only four partners performed a single-stage dissolution test in simulated intestinal medium representing the fed state. Figure 2 illustrates that all partners measured a nearly complete dissolution of indinavir from its formulation $30 \mathrm{~min}$ ( $\mathrm{RSD}=7.6 \%$ ) after the initiation of the dissolution test. Dissolution profiles were highly reproducible among the four participating partners. The high reproducibility in this experiment may be linked to the lack of supersaturation, and thus precipitation: the theoretical maximum concentration of one capsule Crixivan ${ }^{\circledast}(0.8 \mathrm{~g} / \mathrm{L})$ in $500 \mathrm{~mL} \mathrm{FeSSIF}$ is lower than the thermodynamic solubility of indinavir in FeSSIF $(1.36 \mathrm{~g} / \mathrm{L})$ 


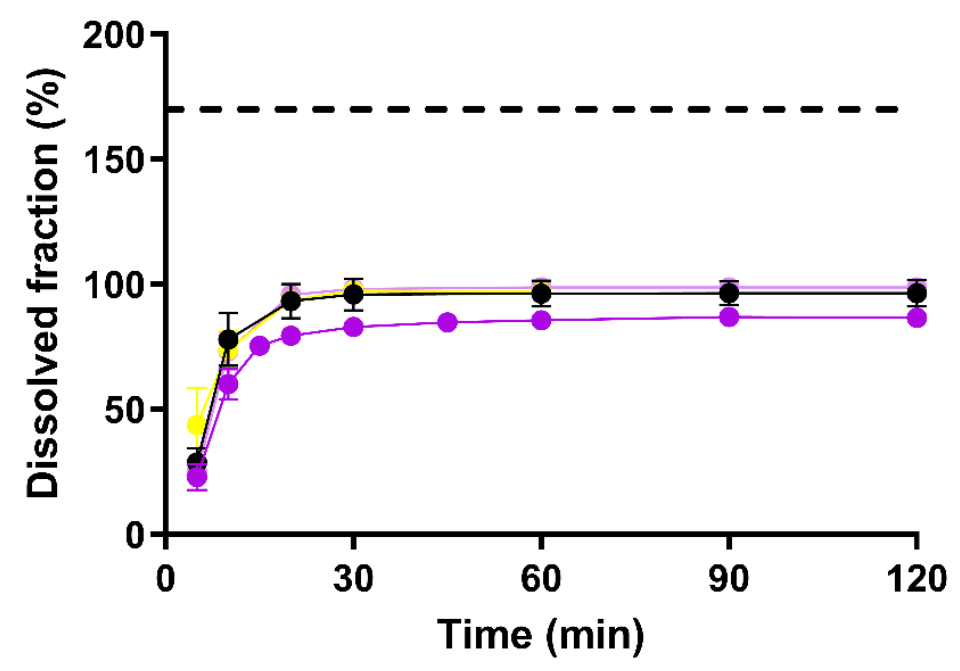

298

Figure 2: Dissolution of indinavir from Crixivan ${ }^{\circledR} 400 \mathrm{mg}$ capsules in FeSSIF. Each colored curve represents the results from one partner (mean $\pm S D, n=3-6)$. The dotted line represents the dissolved fraction corresponding to the thermodynamic solubility of indinavir in FeSSIF.

\subsection{Two-stage dissolution test}

In addition to the single medium dissolution experiments, a two-stage dissolution test was performed simulating the gastrointestinal transfer, i.e. from the acidic environment of the stomach to the neutral environment of the small intestine. The average dissolution profiles for fourteen different partners are illustrated in Figure 3. Since one partner repeated the experiments, fifteen different dissolution profiles were obtained. As previously described (cfr. 3.1.), all partners observed almost complete dissolution of the weak base indinavir in the acidic environment. Upon addition of $250 \mathrm{~mL}$ of $2 x$ FaSSIF (pH 7.5), dissolution profiles diverged. Most partners (13 out of 15) clearly observed supersaturated concentrations of indinavir following the drastic shift in $\mathrm{pH}$; however, the degree and time span of the intestinal supersaturation varied substantially among the participating partners. For instance, two partners observed extensive supersaturation for more than 20 min while two other partners measured extensive intestinal precipitation immediately upon the gastrointestinal transfer. One hour after the $\mathrm{pH}$-shift, all partners measured indinavir concentrations (RSD $=37.5 \%)$ approaching its thermodynamic equilibrium solubility in FaSSIF ( $0.048 \mathrm{~g} / \mathrm{L}$ ) which corresponds to $5.2 \%$ of the administered dose (see Figure 3, dotted line) [13]. 
Even though all partners were provided with standardized protocols to perform the dissolution experiments, interlaboratory reproducibility was relatively poor (e.g. RSD $=64.0 \%$ and $92.5 \%$ at $40 \mathrm{~min}$ and $50 \mathrm{~min}$, respectively), particularly for time points where supersaturation/precipitation (range 30the interlaboratory variability. Moreover, vessel-to-vessel data for each partner were rather consistent suggesting that the protocol was interpreted consistently by each partner but not among partners.

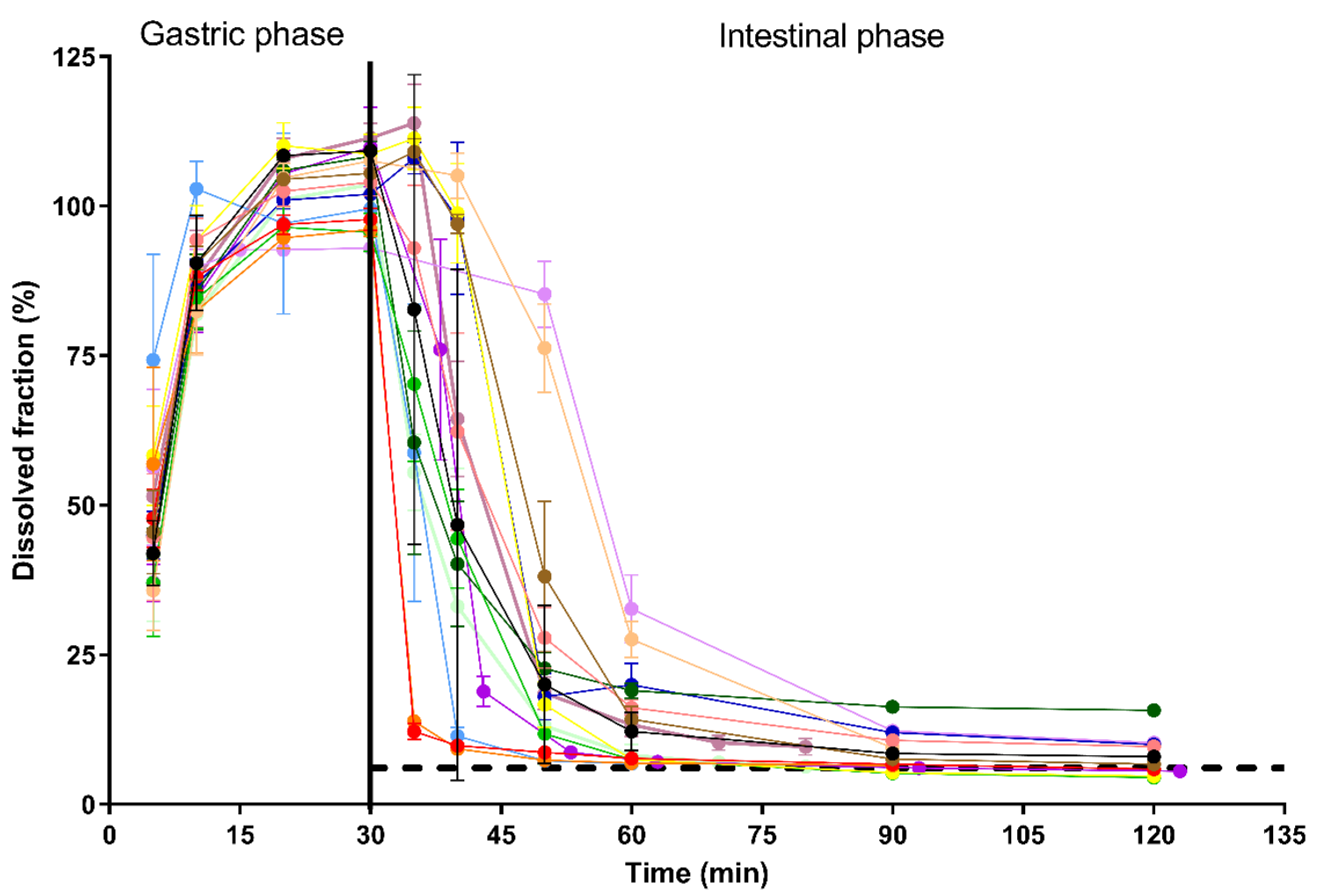

Figure 3: Dissolution of indinavir from Crixivan ${ }^{\oplus} 400 \mathrm{mg}$ capsules in the two-stage dissolution test. Each colored curve represents the results from one partner (mean $\pm S D, n=3-6$ ). The dotted line represents the dissolved fraction corresponding to the thermodynamic solubility of indinavir in FaSSIF.

In human healthy volunteers, intestinal precipitation of indinavir has been observed upon gastrointestinal transfer [12]. The dissolution profiles from the two-stage dissolution test also consistently showed precipitation, but it was generally more pronounced in vitro than in vivo. Since in 
vivo, the supersaturated drug has the choice to precipitate or to permeate across the intestinal epithelium, it is likely that in vivo absorption reduces intestinal supersaturation and subsequently the driving force for precipitation. As a result, when assessing supersaturation in vitro, the precipitated fraction might be overestimated in the absence of an absorptive sink compartment, as is the case for the single medium run in FaSSIF and the two-stage dissolution test using the USP 2 dissolution apparatus.

\subsection{Experimental factors affecting in vitro precipitation}

The relatively high variability in the intestinal dissolution profiles obtained in single- and two-stage dissolution tests (see Figure 1 and 3 ) is most likely related to variations in precipitation from the metastable state of supersaturation. Indeed, dissolution profiles in the media where no supersaturation occurs (FaSSGF in Figure 3, FeSSIF in Figure 2), demonstrate good reproducibility between partners. To better understand supersaturation/precipitation behavior in the USP 2 dissolution apparatus, it is necessary to identify the sources of the interlaboratory variability. Hence, in further experiments, it was investigated whether slight changes in media $\mathrm{pH}$, time of sample dilution and the possibility of heterogeneous nucleation might contribute to the observed variability.

\subsection{1. $\mathrm{pH}$}

When adding $2 x$ FaSSIF of $\mathrm{pH} 7.5$ to FaSSGF with a $\mathrm{pH}$ of 1.6 , the $\mathrm{pH}$ of the final medium is expected to decrease to 6.5 , which is close to the second pKa of indinavir (5.9). Accordingly, a slight deviation from pH 6.5 might significantly change the solubility and dissolution behavior of indinavir, and thus be a potential source of variation. To test this hypothesis, the two-stage dissolution experiment was repeated with $2 x$ FaSSIF buffers containing a pH of 7.3 or 7.7. Using these slightly different buffers, the $\mathrm{pH}$ after the $\mathrm{pH}$-shift amounted to 6.33 and 6.70, respectively. However, the average dissolution profiles appeared in close agreement to the reference experiment which used a 2x FaSSIF buffer with a pH of 7.5, as illustrated in Figure 4. When 2x FaSSIF buffer was adjusted to $\mathrm{pH}$ of 6.5 corresponding to FaSSIF itself, the dissolution/precipitation profile differed substantially, as depicted in Figure 4. In 
this case, the $\mathrm{pH}$ of the final medium (4.4) was much lower than the pKa of indinavir and, due to a marked increase in indinavir solubility at this $\mathrm{pH}(0.94 \mathrm{~g} / \mathrm{L}$ at $\mathrm{pH} 4.5$ compared to $0.048 \mathrm{~g} / \mathrm{L}$ at $\mathrm{pH} 6.5)$, no indinavir precipitation was observed upon the pH-shift (Figure 4, circles). Ultimately, these results suggest that slight differences in starting buffer $\mathrm{pH}$ are not the main source of the variable precipitation. Even so, it is recommended that the $\mathrm{pH}$ of $2 \mathrm{x}$ FaSSIF should be as accurate as possible.

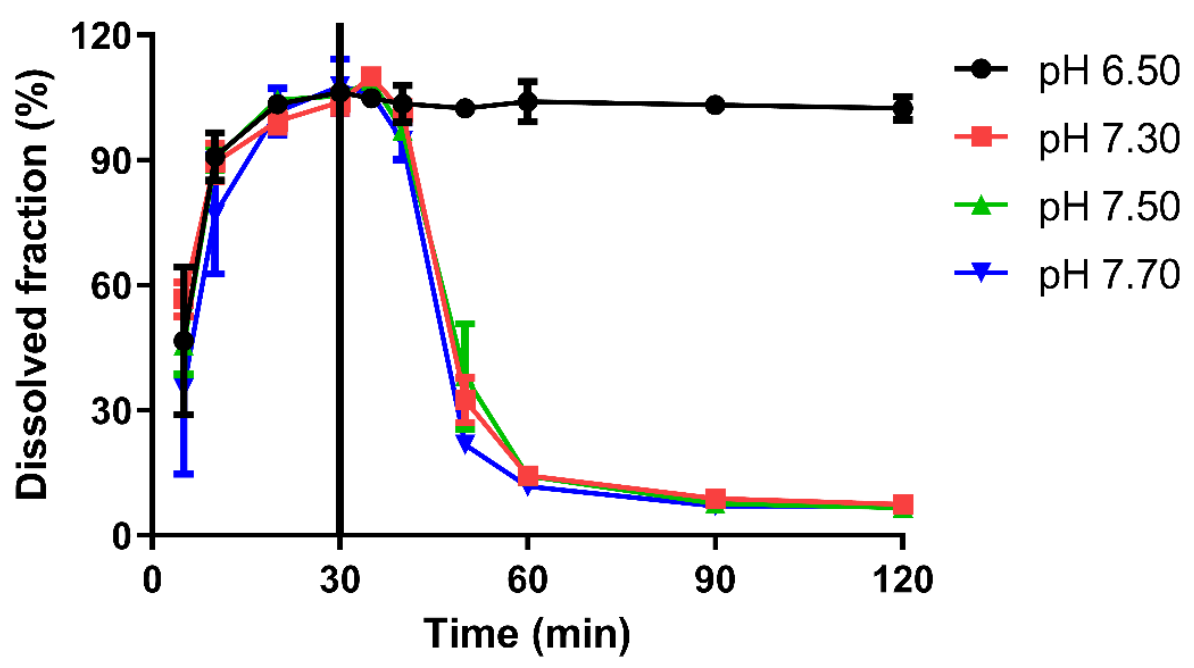

Figure 4: Dissolution of indinavir in the two-stage dissolution test using $2 x$ FaSSIF solutions with a different $p H$. Data are presented as mean $\pm S D, n=3$.

\subsubsection{Sample dilution}

The protocol clearly stated that, if supersaturation is likely to occur (which is the case for indinavir in media simulating the intestinal environment), samples should be diluted immediately after collection to circumvent further precipitation. Nevertheless, post-hoc questioning indicated that the time elapsed between sample collection and dilution appeared to be highly variable among the different partners, as listed in Table 2. Only five partners diluted their samples immediately after collection, as required by the protocol. Five other partners diluted their samples within 10 to $30 \mathrm{~min}$ after sampling, and two partners diluted their samples when the dissolution experiment was complete. Furthermore, one partner did not perform a dilution step and one partner measured indinavir concentrations online, using a semi-automated system. When filtered samples are not diluted immediately after collection, further in-vial precipitation could take place, resulting in an underestimation of the indinavir 
concentration in solution. Therefore, variations in the time span between sample collection and dilution might contribute to the observed interlaboratory variability.

In fact, one partner who observed immediate precipitation upon the $\mathrm{pH}$-shift (Figure 3, orange colored curve) did not perform a dilution step, indicating that precipitation was indeed ongoing in the vial. The other partner who recorded a similar dissolution profile did dilute the samples (Figure 3, red colored curve), but only 15 minutes after sampling rather than immediately.

On the other hand, two partners observed extensive supersaturation for more than 20 min post pHshift (Figure 3, salmon pink and lilac colored curve). One of these partners measured concentrations of indinavir using a semi-automated UV system, so it is possible that variable background UV absorbance due to the presence of precipitated particles interfered with the UV signal. Recently, Jede et al. proposed an in-line derivative spectroscopic method to measure drug concentrations online in highly turbid samples [18]. This methodology could be considered as an interesting alternative as the increased sampling frequency and lack of sample preparation allow better capturing of supersaturation/precipitation.

Table 2. Overview of sample dilution conditions for each partner

\begin{tabular}{cccc}
\hline Partner & Dilution & Length of time to dilute & Preheat \\
\hline 1 & No* & N/A* & Yes \\
2 & Yes & $<30$ min & Yes \\
3 & Yes & Immediate & Yes \\
4 & Yes** & $<15$ min & Yes \\
5 & Yes & Immediate & Yes \\
6 & Yes & Post run completion (<2 hours) & No \\
7 & Yes & Post run completion (<2 hours) & Yes \\
8 & Yes & $<30$ min & Yes \\
9 & Yes & Immediate & Yes \\
10 & Yes & Immediate & Yes \\
11 & Yes & Immediate & Yes \\
12 & Yes & $<10$ min & Yes \\
13 & Yes & $<15$ min & Yes \\
14 & No & N/A & Yes \\
\hline
\end{tabular}


To gain further insight into the impact of the time span between sample collection and dilution,

400 additional experiments were performed in which the filtered samples were diluted at predetermined

401 time points including (i) immediately after sampling, (ii) after each time point, (iii) after the experiment,

402 (iv) 2 hours after the experiment, and (v) 24 hours after the experiment. This experiment revealed that

403 the time elapsed between sampling and dilution greatly affects the obtained dissolution/precipitation

404 profiles of indinavir. As depicted in Figure 5, this effect is more pronounced for samples in which

405 extensive supersaturation (35-50 $\mathrm{min}$ ) was observed. For instance, in samples collected $10 \mathrm{~min}$ after

406 the $\mathrm{pH}$-shift, the dissolved fraction of indinavir decreased $34 \%$ if samples were diluted after the

407 experiment instead of immediately; when filtered samples were only diluted 24 hours after the

408 experiment, a $63 \%$ decrease was observed. Although none of the partners waited 24 hours to dilute

409 their samples, these data suggest that immediate dilution is highly recommended to circumvent

410 further in-vial precipitation in samples with supersaturated concentrations. In-vial precipitation may

411 further cause chromatographic issues including blockage of the column or baseline and retention time

412 variability. This may introduce additional variability due to difficulties in selecting consistent

413 integration parameters. Furthermore, the protocol stated that, upon filtration, any excess in the

414 syringe should be returned to the vessel, thereby potentially inducing precipitation.

415 Overall, these data clearly indicate that it's highly advisable to increase the level of the instructions of

416 the sampling methodology (sample dilution and preparation) in the standard operating protocols. 


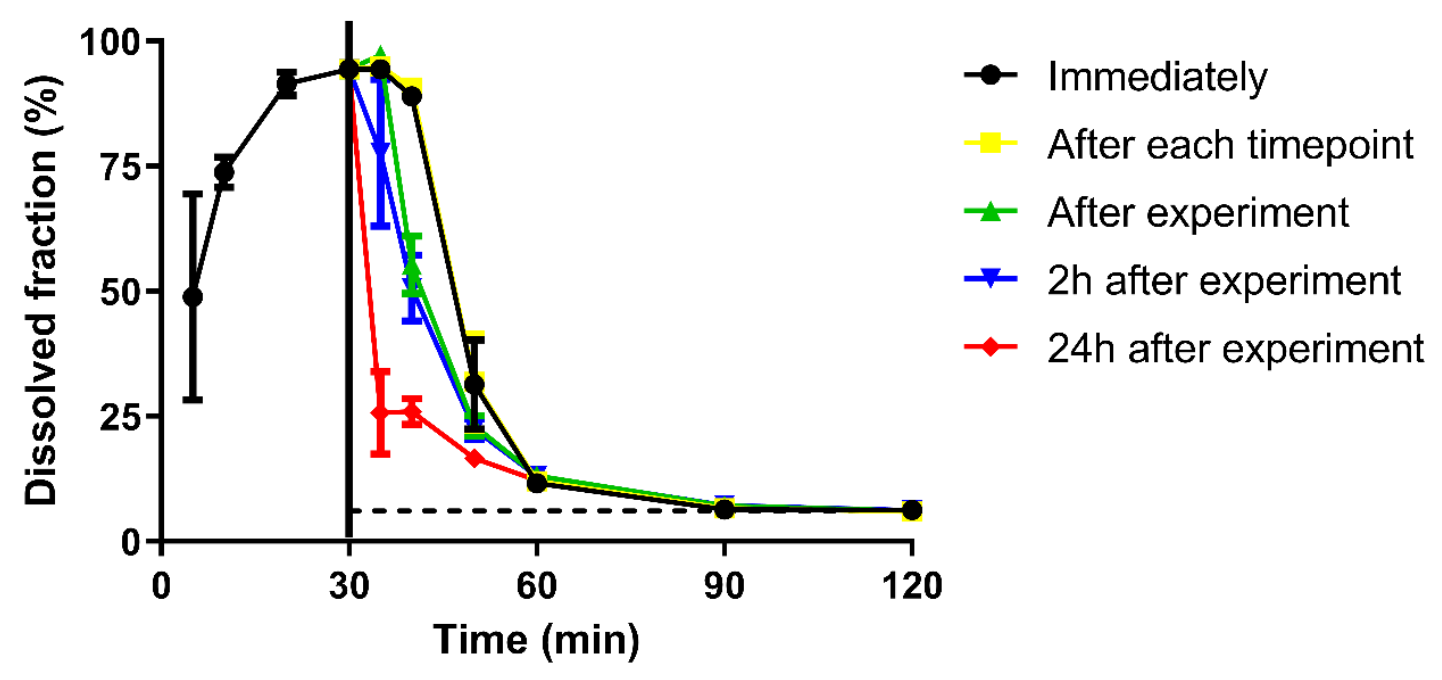

417

418

419

420

421

422

423

424

425

426

427

428

429

430

431

432

Figure 5: Impact of time span to dilute the collected samples on the apparent precipitation behavior of indinavir. Data are presented as mean $\pm S D, n=3$. Dotted line represents the dissolved fraction corresponding to the thermodynamic solubility of indinavir in FaSSIF.

\subsubsection{Heterogeneous nucleation}

The crystal growth theory indicates that undissolved particles or impurities on the surface could induce precipitation by acting as nuclei for crystal growth [19]. We therefore investigated the potential role of heterogeneous nucleation (i.e., nucleation on impurity surfaces) in causing the observed interlaboratory variability.

\subsubsection{Apparatus material}

In the study protocol, the apparatus material for paddles, shafts and sinkers was not explicitly specified, making it a potential source of variability if other materials were used by the different participating partners. For example, it has been reported that PTFE (polytetrafluoroethylene, Teflon) might induce heterogeneous nucleation [20]. To investigate the effect of the apparatus material on the dissolution/precipitation behavior of indinavir, an additional set of experiments was performed by using instruments consisting of PTFE or stainless-steel. The dissolution/precipitation profiles were very similar, as depicted in Figure 6, implying that the type of apparatus material could be excluded as a potential source of variation in the indinavir results. Additionally, it would be useful for future experiments to explore whether different filter materials and/or pressure forces could affect the 
precipitation rate of indinavir. Therefore, when revising the standard operating protocols, specification

438 of the apparatus and filter material may help to achieve improved reproducibility in future 439 experiments.

Besides, it is known that the mechanism of hydrodynamics (shaking vs. stirring) can drastically affect the precipitation rate of supersaturated drugs. Typically, stirring conditions overestimate the intestinal motility while shaking conditions may better resemble the intestinal motility of the fasted state [21]. Indeed, the applied stirring speed helps to overcome the activation energy for nucleation, resulting in a faster and a higher degree of drug precipitation compared to shaking conditions. However, it is difficult to state if shaking conditions will result in an increased/decreased interlaboratory reproducibility. For instance, the homogeneity within the vessel might be decreased, resulting in local concentration gradients and thus a different supersaturation/precipitation event. A comparative study between stirring and shaking conditions could thus be of interest for a future study.

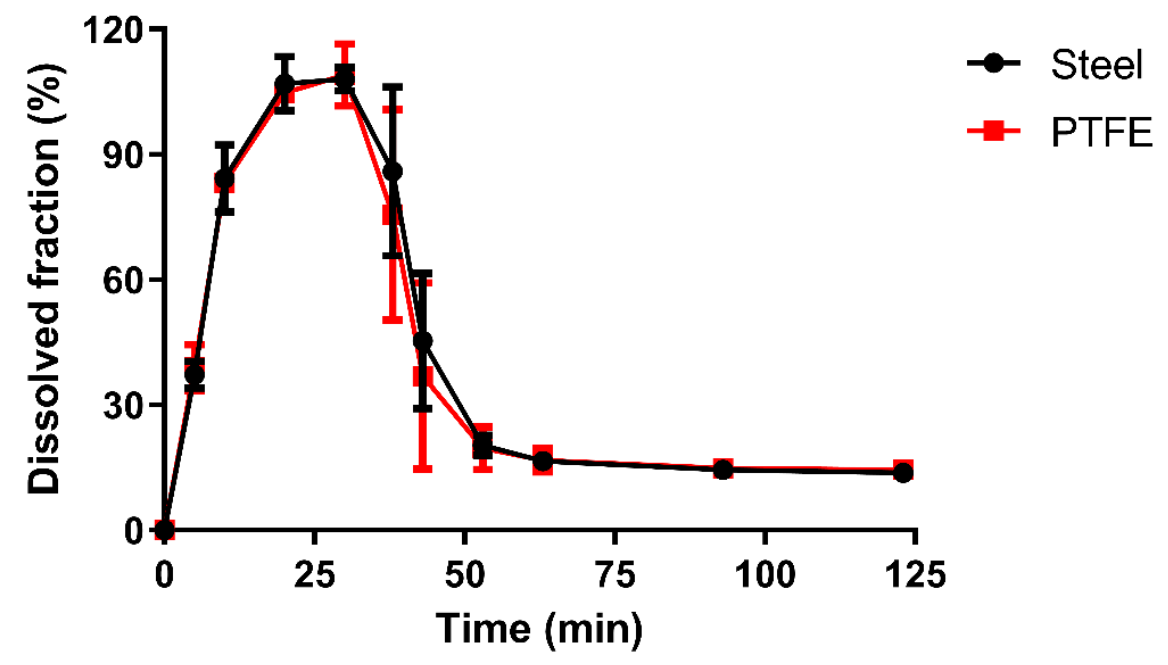

Figure 6: Dissolution of indinavir from Crixivan ${ }^{\oplus} 400 \mathrm{mg}$ capsules in the two-stage dissolution test in which paddles, shafts and sinkers were made either completely of stainless steel or of PTFE. Data are presented as mean $\pm S D, n=3$.

453

\subsubsection{Vessel cleanliness}

In a next step, the impact of undissolved particles, possibly remaining from a previous experiment, on 
quantitatively, the area under the curve of supersaturation (SAUC) was calculated. To this end, the average dissolution profile was plotted along with the thermodynamic equilibrium solubility of indinavir at $\mathrm{pH}$ 6.5, as illustrated in Figure 7. Assuming that the $\mathrm{pH}$ changes immediately from 1.6 to 6.5 upon transfer, the solubility was set at $0.048 \mathrm{~g} / \mathrm{L}$, which is equivalent to $5.2 \%$ of the administered dose [13]. SAUC values were determined using an AUC macro in Microsoft Excel (Figure 7; blue area) for concentrations up to 60 minutes upon $\mathrm{pH}$ shift, as this was the crucial phase of indinavir supersaturation and precipitation.

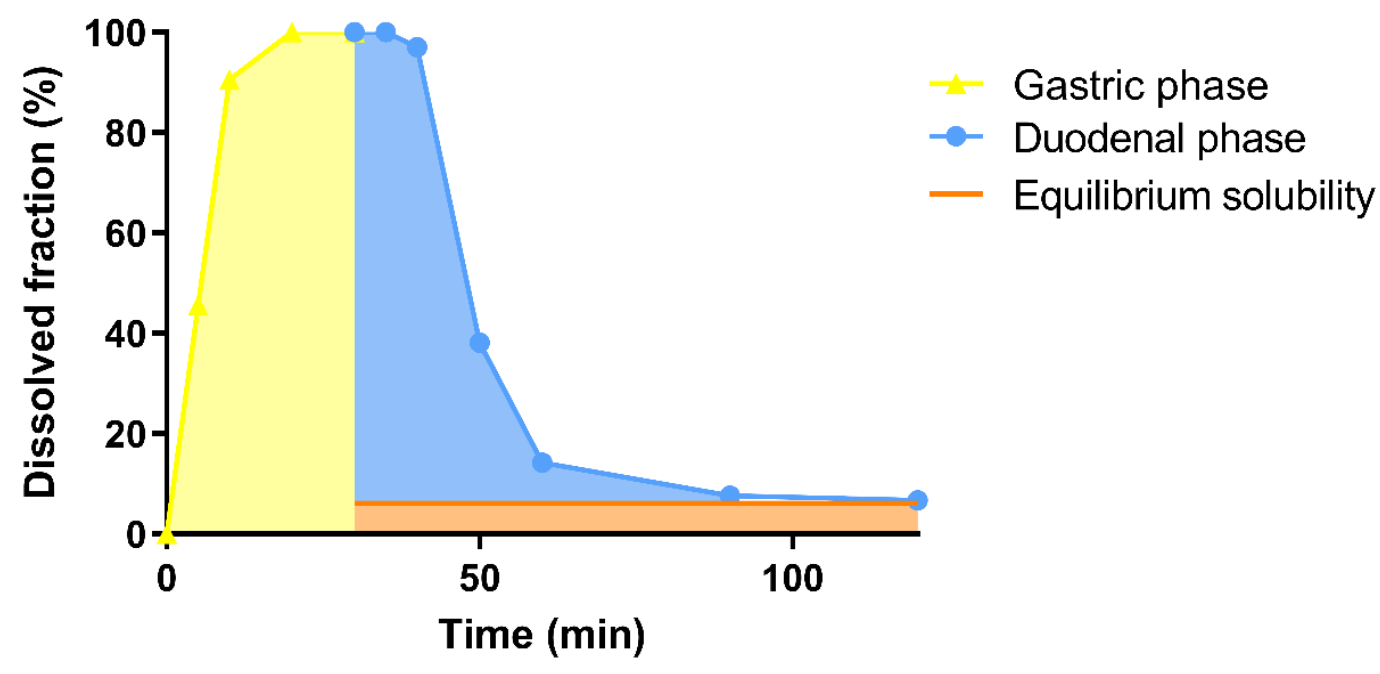

Figure 7: Example graph showing the calculation of SAUC (blue area) for a pH shift dissolution experiment.

Using the same dissolution equipment (Sotax AT7), it was noticed that repetition of the experiment resulted in a decrease of the SAUC with each subsequent experiment, as illustrated in Figure 8 (black bars). The mean SAUC values were determined to be statistically significant $(p<0.05)$ for experiments 2, 3 and 4 compared to experiment 1 and between experiments 2 and 4 . A reason with respect to the higher SAUC value for experiment 1 might have been the naivety of the Sotax AT7 bath to indinavir. Each subsequent experiment had an increased probability of remaining indinavir particles present in the dissolution vessel, which could cause a seeding effect resulting in heterogeneous nucleation. The Sotax AT7 bath is prone to this effect as it utilizes hollow shaft sampling with a coarse mesh on which residues could remain undetected. Furthermore, an additional experiment was executed, in parallel 
with the performance of experiment 4, where 2x FaSSIF was added one hour later than defined in the protocol. It was observed that this prolonged residence time in FaSSGF resulted in a larger SAUC (14.2 instead of 9.8), implying that an increased time in FaSSGF, in which indinavir is highly soluble, might decrease the extent of intestinal precipitation upon $\mathrm{pH}$-shift.

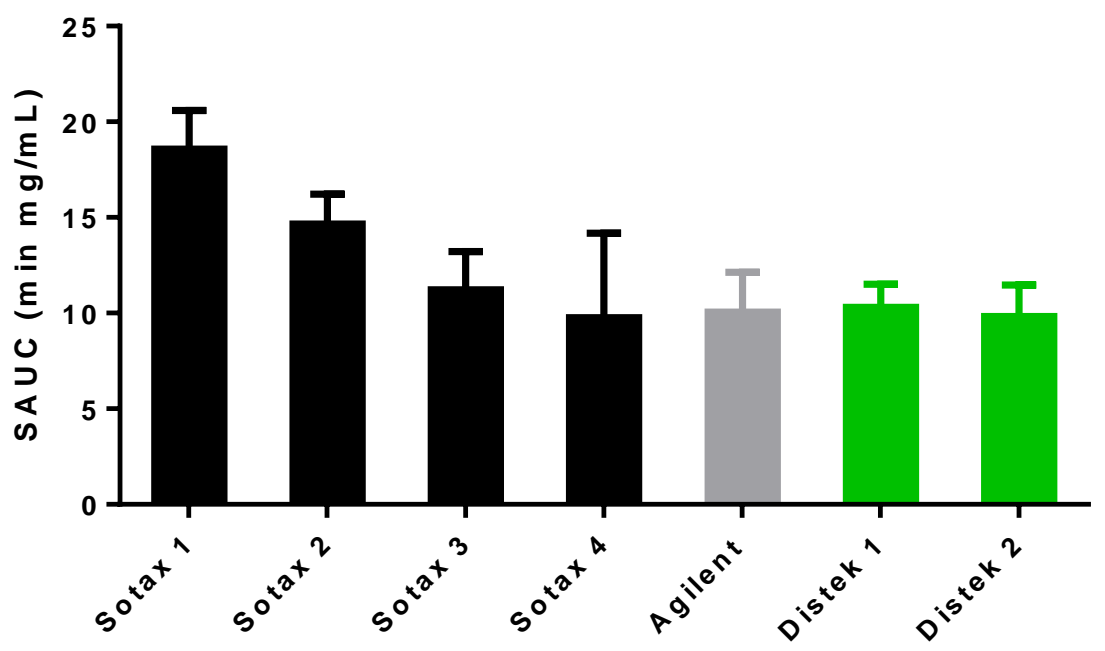

Figure 8: Mean SAUC values for the $\mathrm{pH}$-shift dissolution of indinavir using different brands of USP 2 apparatus including a Sotax AT7, Distek 2100C and Agilent 708-DS bath. All Sotax and Distek replicates were carried out on the same instrument. Data are presented as mean $\pm S D$.

To further investigate the potential nucleation-inducing effect of retained indinavir particles, the twostage dissolution test was repeated using two other types of instruments known to be naïve to indinavir: a Distek $2100 \mathrm{C}$ and an Agilent 708-DS. The SAUC values for both instruments were similar to experiment 3 and 4 for the Sotax AT7 apparatus. Although the Agilent 708-DS was new and therefore naïve for indinavir, it had been calibrated using the prednisone performance verification test (PVT), directly prior to the experiment with indinavir. As the prednisone PVT results in an incomplete dissolution, the presence of residual solid particles, which are not removed during cleaning procedures, could have contributed to heterogeneous nucleation of indinavir. The Distek $2100 \mathrm{C}$ apparatus also generated SAUC values similar to the Agilent 708-DS experiment. Although naïve to indinavir, this apparatus had been used for various other projects. As it is uncommon practice to conduct a wash of the system prior to starting a dissolution in the company which conducted this 
follow-up experiments, heterogeneous nucleation by the presence of undissolved residues and/or dust

497 particles might have induced precipitation.

498 In general, it seems that vessel cleanliness might be a more important factor than naivety to the drug 499 in question with respect to in vitro precipitation. Vessels should be clean and free of retained 500 undissolved particles from previously performed experiments to properly conduct any supersaturation/precipitation experiment. As the protocol did not provide specific cleaning guidelines and differences across institutes exist with respect to the cleaning procedure (solvent, number and

503 frequency of wash cycles), it would be relevant to implement specific cleaning guidelines for 504 dissolution apparatus in the revised protocols to improve the interlaboratory reproducibility of a 505 straightforward precipitation test. Indeed, some partners washed their vessels using tap water while 506 other partners followed an in-house standardized cleaning procedure consisting of different wash 507 cycles using several solvents.

\section{CONCLUSION}

This study describes the results of cross-laboratory biorelevant USP 2 dissolution testing of a commercially available formulation of indinavir, a weak base. Fourteen partners involved in the OrBiTo-consortium participated in these studies. The average dissolution profiles in FaSSGF and FeSSIF were shown to be highly consistent among partners. However, in testing where supersaturation and precipitation occurred, i.e. in FaSSIF (due to the conversion of the salt back to the free base) and in the

514 two-stage test (due to the $\mathrm{pH}$-shift), results were less reproducible. Post-hoc evaluation of various 515 parameters suggested that the reduced reproducibility in the experiments with supersaturation/precipitation was likely attributable to deviations in the protocol with respect to the time elapsed between sample collection and dilution, although other factors may also play a role. To improve the reproducibility of dissolution tests in which supersaturation/precipitation occurs, it's necessary to improve the precision of the instructions in the protocols (e.g. controlled sampling, 
521 as these dissolution tests are widely used within pharmaceutical industry for different research and 522 quality control purposes.

\section{ACKNOWLEDGEMENTS}

524 This work has received support from the Innovative Medicines Initiative Joint Undertaking

525 (http://www.imi.europa.eu) under Grant Agreement No. 115369, resources of which are composed of 526 financial contribution from the European Union's Seventh Framework Program and EFPIA companies' 527 in kind contribution. 


\section{REFERENCES}

[1] E.S. Kostewicz, B. Abrahamsson, M. Brewster, J. Brouwers, J. Butler, S. Carlert, P.A. Dickinson, J. Dressman, R. Holm, S. Klein, J. Mann, M. McAllister, M. Minekus, U. Muenster, A. Müllertz, M. Verwei, M. Vertzoni, W. Weitschies, P. Augustijns, In vitro models for the prediction of in vivo performance of oral dosage forms, Eur. J. Pharm. Sci. 57 (2014) 342-366. doi:10.1016/j.ejps.2013.08.024.

[2] European Medical Agency, 2010, (n.d.).

http://www.ema.europa.eu/docs/en_GB/document_library/Scientific_guideline/2010/01/WC500070039. pdf (accessed April 10, 2018).

[3] A. Ruff, T. Fiolka, E.S. Kostewicz, Prediction of Ketoconazole absorption using an updated in vitro transfer model coupled to physiologically based pharmacokinetic modelling, Eur. J. Pharm. Sci. Off. J. Eur. Fed. Pharm. Sci. 100 (2017) 42-55. doi:10.1016/j.ejps.2016.12.017.

[4] E.S. Kostewicz, M. Wunderlich, U. Brauns, R. Becker, T. Bock, J.B. Dressman, Predicting the precipitation of poorly soluble weak bases upon entry in the small intestine, J. Pharm. Pharmacol. 56 (2004) 43-51. doi:10.1211/0022357022511.

[5] L. Di, B. Feng, T.C. Goosen, Y. Lai, S.J. Steyn, M.V. Varma, R.S. Obach, A perspective on the prediction of drug pharmacokinetics and disposition in drug research and development, Drug Metab. Dispos. Biol. Fate Chem. 41 (2013) 1975-1993. doi:10.1124/dmd.113.054031.

[6] I. Kola, J. Landis, Can the pharmaceutical industry reduce attrition rates?, Nat. Rev. Drug Discov. 3 (2004) 711-715. doi:10.1038/nrd1470.

[7] J. Mann, J. Dressman, K. Rosenblatt, L. Ashworth, U. Muenster, K. Frank, P. Hutchins, J. Williams, L. Klumpp, K. Wielockx, P. Berben, P. Augustijns, R. Holm, M. Hofmann, S. Patel, S. Beato, K. Ojala, I. Tomaszewska, J.-L. Bruel, J. Butler, Validation of Dissolution Testing with Biorelevant Media: An OrBiTo Study, Mol. Pharm. 14 (2017) 4192-4201. doi:10.1021/acs.molpharmaceut.7b00198.

[8] M. Verwei, M. Minekus, E. Zeijdner, R. Schilderink, R. Havenaar, Evaluation of two dynamic in vitro models simulating fasted and fed state conditions in the upper gastrointestinal tract (TIM-1 and tiny-TIM) for investigating the bioaccessibility of pharmaceutical compounds from oral dosage forms, Int. J. Pharm. 498 (2016) 178-186. doi:10.1016/j.ijpharm.2015.11.048.

[9] B. Hens, S.M. Pathak, A. Mitra, N. Patel, B. Liu, S. Patel, M. Jamei, J. Brouwers, P. Augustijns, D.B. Turner, In Silico Modeling Approach for the Evaluation of Gastrointestinal Dissolution, Supersaturation, and Precipitation of Posaconazole, Mol. Pharm. 14 (2017) 4321-4333. doi:10.1021/acs.molpharmaceut.7b00396.

[10] E.S. Kostewicz, L. Aarons, M. Bergstrand, M.B. Bolger, A. Galetin, O. Hatley, M. Jamei, R. Lloyd, X. Pepin, A. Rostami-Hodjegan, E. Sjögren, C. Tannergren, D.B. Turner, C. Wagner, W. Weitschies, J. Dressman, PBPK models for the prediction of in vivo performance of oral dosage forms, Eur. J. Pharm. Sci. Off. J. Eur. Fed. Pharm. Sci. 57 (2014) 300-321. doi:10.1016/j.ejps.2013.09.008.

[11] N. Kaur, A. Narang, A.K. Bansal, Use of biorelevant dissolution and PBPK modeling to predict oral drug absorption, Eur. J. Pharm. Biopharm. Off. J. Arbeitsgemeinschaft Pharm. Verfahrenstechnik EV. 129 (2018) 222-246. doi:10.1016/j.ejpb.2018.05.024.

[12] J. Rubbens, J. Brouwers, J. Tack, P. Augustijns, Gastrointestinal dissolution, supersaturation and precipitation of the weak base indinavir in healthy volunteers, Eur. J. Pharm. Biopharm. Off. J. Arbeitsgemeinschaft Pharm. Verfahrenstechnik EV. 109 (2016) 122-129. doi:10.1016/j.ejpb.2016.09.014.

[13] P. Augustijns, B. Wuyts, B. Hens, P. Annaert, J. Butler, J. Brouwers, A review of drug solubility in human intestinal fluids: implications for the prediction of oral absorption, Eur. J. Pharm. Sci. Off. J. Eur. Fed. Pharm. Sci. 57 (2014) 322-332. doi:10.1016/j.ejps.2013.08.027.

[14] J.H. Lin, I.W. Chen, K.J. Vastag, D. Ostovic, pH-dependent oral absorption of L-735,524, a potent HIV protease inhibitor, in rats and dogs, Drug Metab. Dispos. Biol. Fate Chem. 23 (1995) 730-735.

[15] K.C. Yeh, P.J. Deutsch, H. Haddix, M. Hesney, V. Hoagland, W.D. Ju, S.J. Justice, B. Osborne, A.T. Sterrett, J.A. Stone, E. Woolf, S. Waldman, Single-dose pharmacokinetics of indinavir and the effect of food, Antimicrob. Agents Chemother. 42 (1998) 332-338.

[16] B. Kloefer, P. van Hoogevest, R. Moloney, M. Kuentz, M.L.S. Leigh, J. Dressman, Study of a Standardized Taurocholate- Lecithin Powder for Preparing the Biorelevant Media FeSSIF and FaSSIF, Dissolution Technol. 17 (2010) 6-13.

[17] N. Holmstock, T. De Bruyn, J. Bevernage, P. Annaert, R. Mols, J. Tack, P. Augustijns, Exploring food effects on indinavir absorption with human intestinal fluids in the mouse intestine, Eur. J. Pharm. Sci. Off. J. Eur. Fed. Pharm. Sci. 49 (2013) 27-32. doi:10.1016/j.ejps.2013.01.012. 
[18] C. Jede, C. Wagner, H. Kubas, C. Weber, W. Weitschies, In-line derivative spectroscopy as a promising application to a small-scale in vitro transfer model in biorelevant supersaturation and precipitation testing, J. Pharm. Pharmacol. 70 (2018) 1315-1323. doi:10.1111/jphp.12991.

[19] L. Lindfors, S. Forssén, J. Westergren, U. Olsson, Nucleation and crystal growth in supersaturated solutions of a model drug, J. Colloid Interface Sci. 325 (2008) 404-413. doi:10.1016/j.jcis.2008.05.034.

[20] Bernland, Karin Maria, Nucleating and clarifying polymers, 2010.

[21] S. Carlert, A. Pålsson, G. Hanisch, C. von Corswant, C. Nilsson, L. Lindfors, H. Lennernäs, B. Abrahamsson, Predicting intestinal precipitation - a case example for a basic BCS class II drug, Pharm. Res. 27 (2010) 21192130. doi:10.1007/s11095-010-0213-8.

606 